lizards, small insectivorous birds and seed-eating finches, for instance).

The first step in the analysis consists of logistic regression, for each species and independent variable, of that species: probability of occurrence on an island as a function of the variable. Such regressions are exemplified by graphs of 'incidence' (probability of occurrence) as a function of island area or species number. By an iterative procedure Schoener and Adler extract such species/habitat regressions plus the interactions within each combination of species (that is,

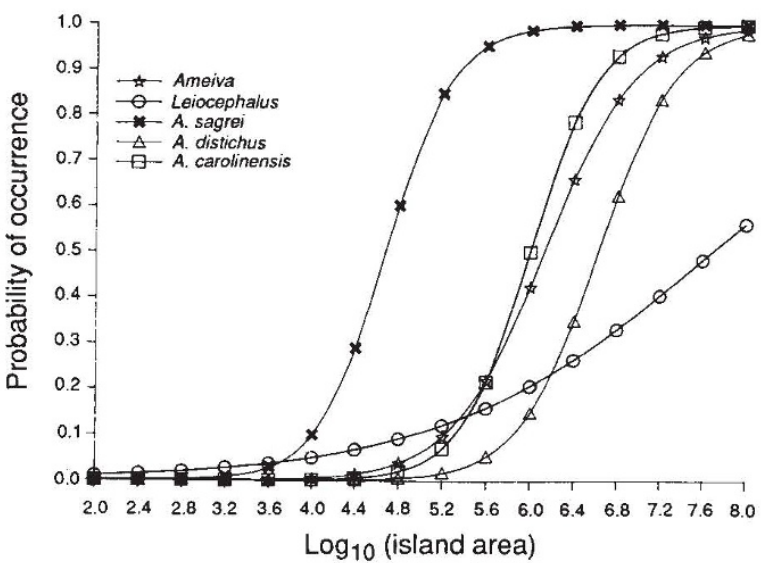

Logistic regression of the proportions of Bahamian islands in a given size class occupied by various lizard species, as a function of $\log _{10}$-transformed island area. That proportion of islands has been termed 'incidence'. The steeper the rise of the curve, the sharper in some sense is the threshold range of island sizes separating islands with and without the named species. (From T. Schoener and G. Adler Am. Nat. 137, 669-692; 1991.)

distributional overlap or avoidance not explained by the species/habitat regressions themselves). The authors stress that the application of this statistical technique to distributional data is new and warrants further testing.

The most striking conclusion is that, after correcting for the effects of habitat variables, the analysis tends to convert positive two-way associations between species (as observed in the raw distributional data) into negative associations. That is, although the direct interaction between the two species is actually negative (they tend to exclude each other), the interaction is masked in the raw data by the similar responses of both species to habitat variables. In effect, the species are enemies doomed to seek out the same types of sites. For instance, to return to the example of two competing duck species, both ducks would be absent from small islands without ponds, both would be present on islands large enough to contain different types of ponds, and only in the range of mediumsized islands with just a single pond would the ducks occupy different islands to the exclusion of each other.

These masking effects of habitat variables prove to be huge. As a result of stripping them away, the proportion of two-way species interactions that are negative increases from 24 to 67 per cent for lizards, and from 3 to 47 per cent for birds. In addition, interactions that are already negative in the raw data become even more strongly so after considering habitat variables.

To interpret the signs of these interactions, Schoener and Adler note that the negative interactions are likely to represent competition, because the species analysed were chosen to be members of the same guild. Examples include wellknown pairs of species that have provided textbook instances of complementary island distributions, such as the mockingbirds Mimus gundlachi and $M$. polyglottos or the lizards Anolis sagrei and the Leiocephalus group. The residual positive interactions may represent parallel responses of species to habitat variables not among those analysed by Schoener and Adler. So two hummingbird species are positively associated both in the raw data and after correction for habitat variables - but probably because distributions of both species are tied to flower abundance, not among the dependent variables measured.

Several other points emerge from the study. Three-way interactions are overwhelmingly negative, reflecting in part the importance of diffuse competition. Negative interactions are more common among lizard species than among bird species, possibly owing to birds' higher immigration rates, hence smaller populations and shorter population lifetimes. Raw habitat variables yield stronger trends than do compound variables produced by principal components analysis.

The main message, however, is that if one wants to test occurrence data for species interactions, one should control for habitat relations because of their huge masking effect. Biogeographers will now be stimulated to reanalyse the many published lists of species occurrences on islands (as well as on islandlike habitat patches) of known area, isolation and elevation. And field workers will be stimulated to measure a greater variety of habitat variables in their studies, for Schoener and Adler have now provided a recipe for taking such measurements into account

Jared $M$. Diamond is in the Department of Physiology, University of California Medical School, Los Angeles, California 90024, USA.

\section{Occult learning}

LAST week Daedalus proposed his 'Skygazer' space telescope. Its forty thousand photodetectors, dividing up the sky between them in fly's-eye fashion, will count all the photons hitting the telescope from the celestial sphere. With appropriate shielding from the Sun, Earth and Moon, it will be exquisitely, ultimately sensitive to the slightest variation of brightness from the rest of the sky.

The Skygazer will be ideal for locating stellar occultations. Daedalus estimates that in a galaxy of ten thousand million stars all wandering around, there must always be at least one star eclipsing another as seen from the Earth: yet (except in the special case of eclipsing binary stars) such an occultation has never been observed. This is not surprising. An occultation is quite undramatic, gives no warning, and cannot last much longer than a day. You would never spot it unless you knew where to look.

The Skygazer spacecraft will tell you. It watches all the sky all the time. Its ultimate photometric accuracy will instantly register the slight loss of light from that section of sky where one star is beginning to hide behind another. The detector whose count is changing will locate the region of loss to within one square degree of sky. An Earth-based Schmidt telescope could then be trained onto that region to find the occultation.

From the changing brightness and spectrum of this joint stellar image, useful information will be gained about the relative velocity, size, brightness, limb darkening and even shape of the protagonists. As the stars then separate, the shifting image of the occulted star as its light is deflected relativistically by the mass of the occulter, will give a measure of that mass. Occultations will be more frequent in some parts of the sky than in others, yielding statistical data on the density and velocity-distribution of stars in various regions of the galaxy.

Best of all, occultation offers about the only possible way of spotting the mysterious 'dark matter' that cosmologists insist must be lying about somewhere. Brown dwarfs, big planets, black clouds and general nonluminous celestial junk, will at last be detectable as they drift in front of luminous stars. If such dark objects have an atmosphere, or even a refractive index, informative lens-effects will further enrich their occultations. And should the long-feared impacting asteroid, or a spacecraft crammed with militant little green men, ever head menacingly towards the Earth, it should reveal itself by occulting background stars long before it could be detected on radar. 